\title{
Walking on Plane and Matrix Square
}

\author{
Balasubramani Prema Rangasamy \\ Former Student of Ramanujan Institute for Advanced Study in Mathematics, University of Madras, Chennai, India \\ Email: balguve@gmail.com
}

How to cite this paper: Rangasamy, B.P. (2021) Walking on Plane and Matrix Square. Advances in Pure Mathematics, 11, 296-316. https://doi.org/10.4236/apm.2021.114019

Received: March 11, 2021

Accepted: April 19, 2021

Published: April 22, 2021

Copyright (c 2021 by author(s) and Scientific Research Publishing Inc. This work is licensed under the Creative Commons Attribution International License (CC BY 4.0).

http://creativecommons.org/licenses/by/4.0/

\begin{abstract}
We know Pascal's triangle and planer graphs. They are mutually connected with each other. For any positive integer $\mathrm{n}, \varphi(n)$ is an even number. But it is not true for all even number, we could find some numbers which would not be the value of any $\varphi(n)$. The Sum of two odd numbers is one even number. Gold Bach stated "Every even integer greater than 2 can be written as the sum of two primes". Other than two, all prime numbers are odd numbers. So we can write, every even integer greater than 2 as the sum of two primes. German mathematician Simon Jacob (d. 1564) noted that consecutive Fibonacci numbers converge to the golden ratio. We could find the series which is generated by one and inverse the golden ratio. Also we can note consecutive golden ratio numbers converge to the golden ratio. Lothar Collatz stated integers converge to one. It is also known as $3 \mathrm{k}+1$ problem. Tao redefined Collatz conjecture as $3 \mathrm{k}-1$ problem. We could not prove it directly but one parallel proof will prove this conjecture.
\end{abstract}

\section{Keywords}

Pascal Triangle, Euler, Twin Prime Conjecture, Goldbach Conjecture, Golden Ratio, Matrix Square, and Collatz Conjecture

\section{Introduction}

In the west, Pascal's triangle appears for the first time in the Arithmetic of Jordanus de Nemore ( $13^{\text {th }}$ century). The binomial coefficients were calculated by Gersonides in the early $14^{\text {th }}$ century, using the multiplicative formula for them. Petrus Apianus (1495-1552) published the full triangle on the frontispiece of his book on business calculations in 1527. Michael Stifel published a portion of the triangle (from the second to the middle column in each row) in 1544, describing it as a table of figurate numbers. In Italy, Pascal's triangle is referred to as Tartaglia's triangle, named for the Italian algebraist Niccolò Fontana Tartaglia (1500-1577), who published six rows of the triangle in 1556. Gerolamo Cardano, 
also, published the triangle as well as the additive and multiplicative rules for constructing it in 1570. Pascal's Traité du triangle arithmétique (Treatise on Arithmetical Triangle) was published in 1655. In this, Pascal collected several results then known about the triangle, and employed them to solve problems in probability theory. The triangle was later named after Pascal by Pierre Raymond de Montmort (1708) who called it "Table de M. Pascal pour les combinaisons" (French: Table of Mr. Pascal for combinations) and Abraham de Moivre (1730) who called it "Triangulum Arithmeticum PASCALIANUM" (Latin: Pascal's Arithmetic Triangle), which became the modern Western name.

Euler's formula states that if a finite, connected, a planar graph is drawn in the plane without any edge intersections, and $v$ is the number of vertices, $e$ is the number of edges and $f$ is the number of faces (regions bounded by edges, including the outer, infinitely large region).

Then as an illustration, in the butterfly graph given above, $v=5, e=6$ and $f=$ 3. In general, if the property holds for all planar graphs of $f$ faces, any change to the graph that creates an additional face while keeping the graph planar would keep $v-e+f$ an invariant. Since the property holds for all graphs with $f=2$, by mathematical induction it holds for all cases. Euler's formula can also be proved as follows: if the graph isn't a tree, then remove an edge that completes a cycle. This lowers both $e$ and $f$ by one, leaving $v-e+f$ constant. Repeat until the remaining graph is a tree; trees have $v=e+1$ and $f=1$, yielding $v-e+f=2$, i.e., the Euler characteristic is 2.

In a finite, connected, simple, planar graph, any face (except possibly the outer one) is bounded by at least three edges and every edge touches at most two faces; using Euler's formula, one can then show that these graphs are sparse in the sense that if $v \geq 3$.

Leonhard Euler introduced the function in 1763. However, he did not at that time choose any specific symbol to denote it. In a 1784 publication, Euler studied the function further, choosing the Greek letter $\pi$ to denote it: he wrote $\pi D$ for "the multitude of numbers less than $D$, and which have no common divisor with it". This definition varies from the current definition for the totient function at $D=1$ but is otherwise the same. The now-standard notation $\varphi(A)$ comes from Gauss's 1801 treatise Disquisitiones Arithmeticae, although Gauss didn't use parentheses around the argument and wrote $\varphi A$. Thus, it is often called Euler's phi function or simply the phi function.

In 1879, J. J. Sylvester coined the term totient for this function, so it is also referred to as Euler's totient function, the Euler totient, or Euler's totient. Jordan's totient is a generalization of Euler's.

The cototient of $n$ is defined as $n-\varphi(n)$. It counts the number of positive integers less than or equal to $n$ that have at least one prime factor in common with $n$.

On 7 June 1742, the German mathematician Christian Goldbach wrote a letter to Leonhard Euler (letter XLIII), in which he proposed the following conjecture:

Every integer that can be written as the sum of two primes can also be written 
as the sum of as many primes as one wishes, until all terms are units.

Goldbach was following the now-abandoned convention of considering 1 to be a prime number, so that a sum of units would indeed be a sum of primes. He then proposed a second conjecture in the margin of his letter, which is easily seen to imply the first:

Every integer greater than 2 can be written as the sum of three primes.

Euler replied in a letter dated 30 June 1742 and reminded Goldbach of an earlier conversation they had had (“...so Ew vormals mit mir communicirt haben...”), in which Goldbach had remarked that the first of those two conjectures would follow from the statement.

Every positive even integer can be written as the sum of two primes.

This is in fact equivalent to his second, marginal conjecture. In the letter dated 30 June 1742, Euler stated:

“Dass ... ein jeder numerus par eine summa duorum primorum sey, halte

ich für ein ganz gewisses theorema, ungeachtet ich dasselbe nicht demonstriren

kann." ("That ... every even integer is a sum of two primes, I regard as a com-

pletely certain theorem, although I cannot prove it.”)

Each of the three conjectures above has a natural analog in terms of the modern definition of a prime, under which 1 is excluded. A modern version of the first conjecture is:

Every integer that can be written as the sum of two primes can also be written as the sum of as many primes as one wishes, until either all terms are two (if the integer is even) or one term is three and all other terms are two (if the integer is odd).

A modern version of the marginal conjecture is:

Every integer greater than 5 can be written as the sum of three primes.

And a modern version of Goldbach's older conjecture of which Euler reminded him is:

Every even integer greater than 2 can be written as the sum of two primes. German mathematician Simon Jacob (d. 1564) noted that consecutive Fibonacci numbers converge to the golden ratio; this was rediscovered by Johannes Kepler in 1608. The first known decimal approximation of the (inverse) golden ratio was stated as "about 0.6180340 " in 1597 by Michael Maestlin of the University of Tübingen in a letter to Kepler, his former student. The same year, Kepler wrote to Maestlin of the Kepler triangle, which combines the golden ratio with the Pythagorean theorem.

Kepler said of these: geometry has two great treasures: one is the theorem of Pythagoras, the other the division of a line into extreme and mean ratio. The first we may compare to a mass of gold, the second we may call a piece of precious jewelry.

The Collatz conjecture is a conjecture in mathematics that concerns a sequence defined as follows: start with any positive integer $n$. Then each term is obtained from the previous term as follows: if the previous term is even, the next 
term is one-half of the previous term. If the previous term is odd, the next term is 3 times the previous term plus 1 . The conjecture is that no matter what value of $n$, the sequence will always reach 1 .

The conjecture is named after Lothar Collatz, who introduced the idea in 1937, two years after receiving his doctorate. It is also known as the $3 n+1$ problem, the $3 n+1$ conjecture, the Ulam conjecture (after Stanisław Ulam), Kakutani's problem (after Shizuo Kakutani), the Thwaites conjecture (after Sir Bryan Thwaites), Hasse's algorithm (after Helmut Hasse), or the Syracuse problem. The sequence of numbers involved is sometimes referred to as the hailstone sequence or hailstone numbers (because the values are usually subject to multiple descents and ascents like hailstones in a cloud), or as wondrous numbers.

Paul Erdős said about the Collatz conjecture: "Mathematics may not be ready for such problems.” He also offered $\$ 500$ for its solution. Jeffrey Lagarias stated in 2010 that the Collatz conjecture "is an extraordinarily difficult problem, completely out of reach of present-day mathematics."

I obtained a history of mathematical concepts and basic concepts of various conjectures from the following references [1]-[9].

In this paper, let us see the generalization of Euler's planer graph formula by Pascal's triangle. Then we find some numbers which would not be the value of any $\varphi(n)$. After that, we see, how six numbers only being the distance between any two consecutive twin prime pair and why the length is always two. Further we see the Division between two even numbers, and what we get a quotient. Is it odd or even? Then, let us show proof of Gold Bach conjecture by some ideological proofs. Then, let us generate a Fibanacci's series like series and find its converge. Finally, let us introduce Matrix square concept then show which would be the parallel proof for Collatz conjecture and Collatz-Tao conjecture.

\section{Vertices and Edges on Graph}

We know, in planer graph, an edge draws between two vertices. How will we draw an edge on isometric (cubical box) volume? So let us define edge on various " $n$ " dimensional volumes.

An edge means, a certain line connecting all vertices without pause.

A certain line connecting three vertices in three dimensional boxes is called an edge of cubic graph.

Or
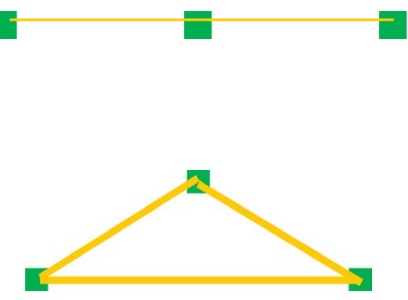

A certain line connecting four vertices in four dimensional boxes is called an 
edge of tesseractic graph.

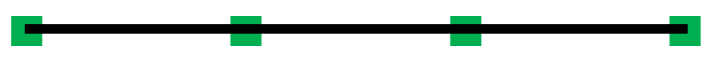

Or

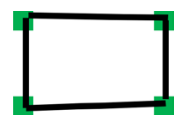

By this way we can define, a certain line connecting " $n$ " vertices ( $n^{\text {th }}$ polygonal) in $\mathrm{n}^{\text {th }}$ dimensional box is called an edge of $n^{\text {th }}$ cubic graph. In below,

1) Figure 1 is the calculation of how many vertices, edges, faces, etc. on a line graph.

2) Figure 2 is the calculation of how many vertices, edges, faces, etc. on a planer graph.

3) Figure 3 is the calculation of how many vertices, edges, faces, etc. on a cubic graph.

4) Figure 4 is the calculation of how many vertices, edges, faces, etc. on a tesseractic graph.

\section{From the above Definitions We Concluded That}

1) Line is the edge on planer graphs

2) Triangle is the edge of cubic graphs

3) Square is the edge of tessaractic graphs

4) By this way, $n^{\text {th }}$ polynomial is the edge of $n^{\text {th }}$ cubic graph.

For two dimensional graphs, one dimensional line is edge. For three dimensional graphs, two dimensional lines are edge! But we never draw a two dimensional line as edge. So only we use a triangle as the edge of cubic graphs. Because a triangle is the minimal closed shape of two dimensional figures.

For tessactic graph, we use square as the edge.

Tetrahedron is also cubic shape but no one connect all vertices continuously.

No one construct a polyhedron lesser than four vertices and or six edges. Suppose $\mathrm{p}$ be a polyhedron with $\mathrm{n}$ vertexes then by a single dipped drawing (without taking hand) we may not connect all vertexes by edges. The maximum number of undrawable edges are $(n-2) / 2$, for even number of vertexes and $(n-3) / 2$, for odd number of vertexes.

Generally above three vertices, no one connect all vertices without pause in polyhedrons.

We know binomial element has two variants. Let us use binomial expansion for planner graphs. If $x=1$ means, graph on line; If $x=2$ means, graph on plane; If $x=3$ means, graph on isometric.

\section{Invariants of $x$-Elements n-Dimensional Cube}

From the above triangles, we concluded that 


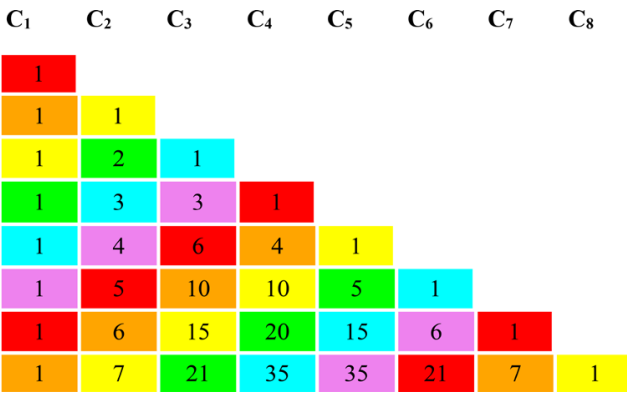

Figure 1. Graph on a Line: $\mathrm{x}=1$.

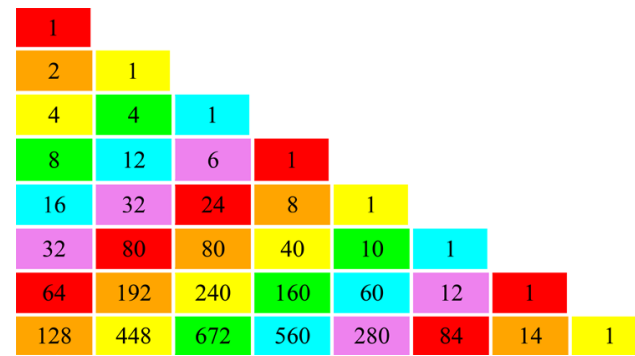

Figure 2. Graph on a Plane. i.e. $\mathrm{x}=2$.

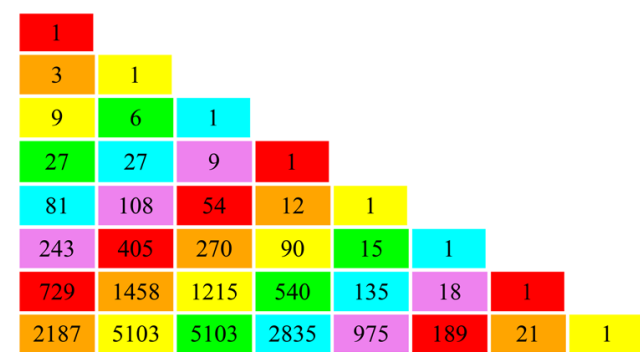

Figure 3. Graph on an Isometric. i.e. $\mathrm{x}=3$.

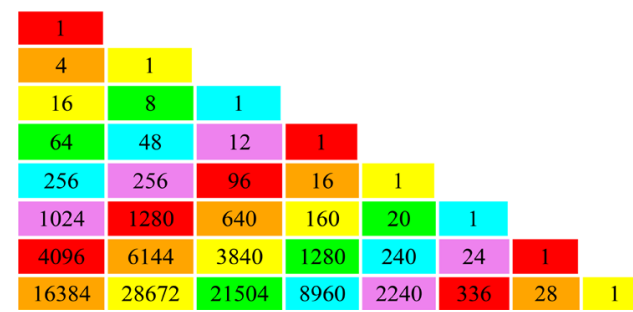

Figure 4. Graph on a Tessaract Cube. i.e. $\mathrm{x}=4$.

Sum of odd column elements of $\sum_{i=0}^{n} a_{i} x^{i}$ - sum of even column elements of $\sum_{i=0}^{n} a_{i} x^{i}=(x-1)^{n}$

$$
\text { i.e. } \sum_{O} \sum_{i=0}^{n} a_{i} x^{i}-\sum_{E} \sum_{i=0}^{n} a_{i} x^{i}=(x-1)^{n},
$$

where is $x$ isgraphing domain.

$$
\text { At } x=1 \quad: 1,0,0,0 \ldots \quad=0^{n} \text {; }
$$




$$
\begin{array}{lll}
\text { At } x=2 & : 1,1,1,1 \ldots & =1^{n} ; \\
\text { At } x=3 & : 1,2,4,8 \ldots & =2^{n} ; \\
\text { At } x=4 & : 1,3,9,27 \ldots & =3^{n} ;
\end{array}
$$

If we left $\left(\begin{array}{l}n \\ 0\end{array}\right)$ elements in $\sum_{O} \sum_{i=0}^{n} a_{i} x^{i}-\sum_{E} \sum_{i=0}^{n} a_{i} x^{i}$ then

$$
\begin{array}{ll}
\text { At } x=1 & : 0,1,-1,1,-1,1 \ldots \\
\text { At } x=2 & : 0,2,0,2,0,2 \ldots \\
\text { At } x=3 & : 0,3,3,9,15,33 \ldots \\
\text { At } x=4 & : 0,4,8,28,80,244 \ldots
\end{array}
$$

Comparing (1) and (2) we concluded that

\section{On odd rows:}

Sum of odd column elements - a sum of even column elements $=$ difference +1

\section{On even rows:}

Sum of odd column elements - a sum of even column elements $=$ difference -1

\section{Generalized Euler's Theorem for Planar Graph}

Preposition 2: Let G be a connected planar graph (2 joints) without $\left(\begin{array}{l}n \\ 0\end{array}\right)$ elements then,

For odd-dimensional $k$ cubes:

Sum of odd column elements - a sum of even column elements $=2$

For even-dimensional $k$ cubes:

Sum of odd column elements - a sum of even column elements $=0$

Ex 1: Let we consider 3 dimensional cube, which is a planner graph, and has 8 vertices, 12 edges and 1 face, then from Figure 2 plane graph, in $4^{\text {th }}$ row, we have $C_{1}=8, C_{2}=12, C_{3}=6$, because of theorem we omitted $C_{4}=1$.

Now $V-E+F=8-12+6=14-12=2$.

Preposition 3: Let $\mathrm{K}$ be $n$-dimensional cube with $\left(\begin{array}{l}n \\ 0\end{array}\right)$ elements then

Sum of odd column elements - sum of even column elements $=(x-1)^{n}$ where " $x$ " is a graphing domain.

\section{Facts 1}

1) For any $n>2$, we get $\varphi[x]$ is always an even number.

2) $\varphi[E] \leq \frac{E}{2}$ is always even number, where $E$ is an even number. 
3) We never find a number $n \in I$, which gives $2(1+6 x) \mid x \in I$ numbers when we find $\varphi[n]$.

$38+30 x \mid x \in I$ also not be the values for $\varphi[n]$.

i.e. $38 \begin{array}{llllllllllllll}68 & 98 & 158 & 188 & 248 & 278 & 308 & 338 & 398 & 428 & 458 & 488 & 518 & 548\end{array}$

$\begin{array}{lllllllllll}578 & 608 & 638 & 668 & 698 & 728 & 758 & 788 & 818 & \ldots\end{array}$

In above numbers some numbers like $38+30 \times 3=38+90=128,368 \ldots$ would be value of $\varphi[n]$, even though their digit sum would be 2 and 8 .

$114,318,298$, would not be a value of $\varphi[n]$, contrarily their digit sum values are $3,1 \ldots$

If digit sum 2, 5 and 8 numbers mostly would not be the value of $\varphi[n]$.

\section{Twin Primes}

$(5,7),(11,13),(17,19),(29,31),(41,43),(59,61) \ldots$ twin primes. In which first element of bracket is lower twin prime and last element of bracket is upper twin prime.

We can generate twin prime by $(6 x-1,6 x+1)$.

In above formula we get odd composite number also. Let we call such numbers as supporter primes.

$(6 x-1,6 x+1)$ for $x=1,2 \ldots$ we get $(5,7),(11,13),(17,19),(23,25),(29,31)$, $(35,37),(41,43),(51,53),(59,61) \ldots$

Where $25,35,51 \ldots$ are called prime supporter of twin prime numbers.

Why the pair $(6 x-1,6 x+1)$ givestw in primes?

Let we jump to Euler's totient function!

We have already seen that there are so many numbers which are not the value for Euler's totient function ever!

General formula of such numbers is: $2(6 x+1)$

$$
\text { i.e. } \varphi[n] \neq 2(6 x+1)
$$

We know totient value of prime number is prime number -1 .

$$
\text { i.e. } \varphi[p]=p-1
$$

Generally for any $p$ and the certain value of any $x$, we can write above formula as

$$
\varphi[p]=2(6 x-1)
$$

Let we see some value of $2(6 x-1)$, for $x=1,2,3 \ldots$ we get

$$
\begin{gathered}
\varphi[11]=10=2 \times 5 \\
\varphi[23]=22=2 \times 11 \\
\varphi[47]=46=2 \times 23 \\
\varphi[59]=58=2 \times 29 \\
\varphi[83]=82=2 \times 41 \ldots \text { in this calculation " } 2 \text { " is the multiplier }
\end{gathered}
$$

and $5,11,23,29,47 \ldots$ are lower twin primes and factors of primes totient values. 
Let we see some value of $2(6 x+1)+1$, for $x=1,2,3 \ldots$ we get

$$
\begin{aligned}
& 14+1=15=3 \times 5 \\
& 26+1=27=3 \times 9 \\
& 38+1=39=3 \times 13 \\
& 50+1=51=3 \times 17 \\
& 62+1=63=3 \times 21 \\
& 74+1=75=3 \times 25 \ldots \text { in this calculation " } 3 \text { " is the multiplier and } \\
& 14,26,50,62,74 \ldots \text { are negated totient values. }
\end{aligned}
$$

In the above calculations, 2 and 3 are plays important role. Twin primes are given by $(6 x-1,6 x+1)$, we could say $6 x-1 \equiv 6 x+5 \bmod 6$. Two and three are additive factors of five and multiplicative factors of six and also difference of $(3,2)=1$.

Length metric of the $(6 x-1,6 x+1)$ is always two.

$$
\text { i.e. }|6 x+1-(6 x-1)|=|6 x+1-6 x+1|=2 \text {. }
$$

So the only distance of twin primes is always two.

So we concluded that generators of twin primes are constructed by $1,2,3,5$, and 6. So only we say the pair $(6 x-1,6 x+1)$ givestwin primes.

Preposition 4: Let $x \geq 2$ be the integer then $\varphi[\varphi[\varphi[\cdots \varphi[x]]]]=1$. i.e. $\varphi^{n}[x]=1$. (15)

Where $n$ is the totient order of $x$.

Proof: Let $x$ be any number then $x>\varphi[x]$.

Let we take $\varphi[x]=x_{1}$ then $x_{1}>\varphi\left[x_{1}\right]$

By this way, we can obtain the totatives, $\varphi[x]>\varphi\left[x_{1}\right]>\varphi\left[x_{2}\right]>\cdots>\varphi[2]=1$

Ex 2:

1) Let $x=693$ then $693>\varphi[693]=360$

$$
\begin{gathered}
360>\varphi[360]=96=\varphi^{2}[693] \\
96>\varphi[96]=32=\varphi^{3}[693] \\
32>\varphi[32]=16=\varphi^{4}[693] \\
16>\varphi[16]=8=\varphi^{5}[693] \\
8>\varphi[8]=4=\varphi^{6}[693] \\
4>\varphi[4]=2=\varphi^{7}[693] \\
2>\varphi[2]=1=\varphi^{8}[693]
\end{gathered}
$$

Totient order of 693 is 8

\section{Facts 2}

1) If $\operatorname{GCD}(a, b)=k$ then $\operatorname{GCD}\left(a^{n}, b^{n}\right)=k^{n}$, where $k$, and $n \in Z$.

2) If $\operatorname{GCD}(a, b)=k$ and $\frac{a}{k}=c ; \frac{b}{k}=d$ then 
$\operatorname{GCD}\left(a^{n}, b^{n}\right)=k^{n}$ and $\frac{a^{n}}{k^{n}}=c^{n} ; \frac{b^{n}}{k^{n}}=d^{n}$, where $c, d, k$, and $n \in Z$.

3) We can generalize above as If $\operatorname{GCD}\left(a_{1}, a_{2}, a_{3}, \cdots, a_{i}\right)=k$ and

$\frac{a_{1}}{k}=b_{1}, \frac{a_{2}}{k}=b_{2}, \frac{a_{3}}{k}=b_{3}, \cdots, \frac{a_{i}}{k}=b_{i}$ then $\operatorname{GCD}\left(a_{1}^{n}, a_{2}^{n}, a_{3}^{n}, \cdots, a_{i}^{n}\right)=k^{n}$ and

$\frac{a_{1}^{n}}{k^{n}}=b_{1}^{n}, \frac{a_{2}^{n}}{k^{n}}=b_{2}^{n}, \frac{a_{3}^{n}}{k^{n}}=b_{3}^{n}, \cdots, \frac{a_{i}^{n}}{k^{n}}=b_{i}^{n}$, where $i, k$ and $n \in Z$.

4) If $p_{1} p_{2} p_{3} \cdots p_{i}$ are distinct primes, then $p_{1}^{a} \neq p_{2}^{b} \neq p_{3}^{c} \neq \cdots \neq p_{i}^{\alpha}$.

5) We can write any composite number as the product of prime numbers. i.e. $c=p_{1}^{a} p_{2}^{b} p_{3}^{c} \cdots$

Preposition5: Let $a, b, c$ are composite positive integers and $a+b=c$, also $k=\operatorname{GCD}(a, b, c)$ then $x+y=z$ is relatively prime with each other, where $x=\frac{a}{k} ; y=\frac{b}{k} ; z=\frac{c}{k}$.

\section{Proof:}

Let $a, b, c$ are composite positive integers and $a+b=c$, also $k=\operatorname{GCD}(a, b, c)$ then we can write $a+b=c$ as $x k+y k=z k$.

If $\operatorname{GCD}\left(a_{1}, a_{2}, a_{3}, \cdots, a_{i}\right)=1$, we multiply each element of $\operatorname{GCD}\left(a_{1}, a_{2}, a_{3}, \cdots, a_{i}\right)=1$ with $k$, we get $\operatorname{GCD}\left(k a_{1}, k a_{2}, k a_{3}, \cdots, k a_{i}\right)=k$, which means all the relatively prime numbers changed into composite number of $k$.

So we divide, $x k+y k=z k$ by $k$.

Hence we get $x+y=z$

Preposition6: Let $a, b, a+b$ are composite positive integers with GCD $k$, then we can find $a^{n}+b^{n}=(a+b)^{n}-\sum_{i=1}^{n-1}\left(\begin{array}{l}n \\ i\end{array}\right) a^{n-i} b^{i}$ with GCD $k^{n}$.

If we divide $a^{n}+b^{n}=(a+b)^{n}-\sum_{i=1}^{n-1}\left(\begin{array}{l}n \\ i\end{array}\right) a^{n-i} b^{i}$ by $k^{n}$, we get $x+y=z$ with GCD 1,

$$
\text { Where } x=\frac{a^{n}}{k^{n}} ; y=\frac{b^{n}}{k^{n}} \text { and } z=\frac{c^{n}-\sum_{i=1}^{n-1}\left(\begin{array}{l}
n \\
i
\end{array}\right) a^{n-i} b^{i}}{k^{n}}=\frac{a^{n}+b^{n}}{k^{n}} \text {. }
$$

\section{Definition: Residue Factors}

Let $A$ be a dividend, its factors are $a b c d$ and $B$ be a divisor, its factors are $a b c$ then the residue factor of $A \div B$ is $d$.

If $d$ is even then the residue factor is even, else it is odd. i.e.

If $d \equiv 0(\bmod 2), d$ is even;

If $d \equiv 1(\bmod 2), d$ is odd.

Ex 3:

1) Let $A=48$ and $B=16$ then factors of $A=2^{4} \times 3$ and factors of $B=2^{4}$ then residue factor $=\frac{2^{4} \times 3}{2^{4}}=3$. The residue factor is odd. 
2) Let $A=210$ and $B=14$, then

factors of $A=2 \times 3 \times 5 \times 7$ and Factors of $B=2 \times 7$ then residue factor $=\frac{2 \times 3 \times 5 \times 7}{2 \times 7}=3 \times 5=15$. The residue factor is odd.

3) Let $A=48$ and $A=24$, then

factors of $A=2^{4} \times 3$ and Factors of $B=2^{3} \times 3$ then residue factor $=\frac{2^{4} \times 3}{2^{3} \times 3}=2$.

The residue factor is even.

Division

$O \div O=O$ or $d$

$E \div O=E$ or $d$

$E \div O \neq O$ because we never get $E \neq O^{2}$;

$E \div E=O$ if the residue factor is odd or $d$;

$E \div E=E$ if residue factor is even or $d$, where $d$ is decimal value. *

\section{Gold Bach Conjecture}

Every even integer greater than two can be expressed as the sum of two prime numbers.

Proof:

We know $4=2+2,6=3+3,8=3+5,10=5+5=7+3,12=7+5,14=7+$ $7,16=5+11$ but is it true for all even numbers? So, we try to prove every even integer greater than two can be expressed as the sum of two prime numbers by some ideological concepts. We know the fact four only expressed by the sum of two even prime. In other words, no even integer greater than four can be expressed by the sum of two even prime. But it can be expressed by two odd prime.

Let $E$ be an even number and $O_{1}$ and $O_{2}$ are odd numbers.

We can express $E$ as sum of two odd numbers. i.e. $E=O_{1}+O_{2}$.

Using the above facts, we can say all odd prime numbers are the members of odd numbers, i.e. $p \subset O$.

Every prime number can be expressed as the sum of an odd number and even number. Also, we can express the prime number as the difference of an odd number and even number.

$$
\text { i.e. } o_{i}+e_{i}=p_{i} \in O \text { and } o_{j}-e_{j}=p_{j} \in O \text {. }
$$

where $i, j \in I$

$$
\text { i.e. } o_{1}+e_{1}=p_{1} \in O_{1} \text { and } o_{2}-e_{2}=p_{2} \in O_{2} \text {. }
$$

Hence, we can express

$$
E=\left(O_{1}+O_{2}\right)=\left(\left(o_{1}+e_{1}\right)+\left(o_{2}-e_{2}\right)\right)=p_{1}+p_{2} .
$$

More precisely, we can express above as,

$$
\begin{aligned}
& E=\left(O_{1}+O_{2}\right)=\left(\left(o_{1}+e_{1}\right)+\left(o_{2}+e_{2}\right)\right)=p_{1}+p_{2} \\
& E=\left(O_{1}+O_{2}\right)=\left(\left(o_{1}-e_{1}\right)+\left(o_{2}-e_{2}\right)\right)=p_{1}+p_{2}
\end{aligned}
$$




$$
E=\left(O_{1}+O_{2}\right)=\left(\left(o_{1}+e\right)+\left(o_{2}-e\right)\right)=p_{1}+p_{2}
$$

Ex 4:

Let 94, we can express $94=63+41=25+69=37+57=\ldots$ for instance,

1) Let 63 and 41, its sum is 94.94 is an even number. Four ended number. The possibility of summation is $1+3,5+9$, and $7+7.63$ and 41 are odd numbers, but 63 is a composite number, and 41 is a prime number. We need one prime number instead of 63 . We know prime number 61 is near to 63 . But $61+2$ is 63 , so we subtract two from 63 . Now we get 61 . To balance equality of sum, we should add the same 2 with 41 . Now we get 43 and 43 is a prime. Also, we get 1 and 3 combinations. So we can express $94=(63-2)+(41+2)=61+43$.

2) Let 25 and 69, its sum is 94.94 is an even number. Four ended number. The possibility of summation is $1+3,5+9$, and $7+7.25$ and 69 are odd numbers but both are composite numbers; we need two prime numbers instead of 25 and 69 . We know the prime number $\{\ldots 17,19,23,29,31,37 \ldots\}$ is near to 25 . Also $\{\ldots 57,61,67,71,73 \ldots\}$ is near to 69 . Select:

If $e=2$ then

$$
94=(25+69)=((25-2)+(69+2))=23+71,
$$

$e=2$ opts for this way of summation.

$$
\text { But } 94=(25+69)=((25+2)+(69-2))=27+67 \text {, }
$$

$e=2$ is not suited for this way of summation.

If $e=6$ then $94=(25+69)=((25-6)+(69+6))=19+75, e=6$ is not suited for this.

If $e=6$ then $94=(25+69)=((25+6)+(69-6))=31+64, e=6$ is not suited for this.

If $e=8$ then $94=(25+69)=((25+8)+(69-8))=33+61, e=8$ is not suited for this.

If $e=8$ then $94=(25+69)=((25-8)+(69+8))=17+77, e=8$ is not suited for this.

If $e=16$ then

$$
94=(25+69)=((25-16)+(69+16))=9+85,
$$

$e=16$ is not suited for this way of summation.

$$
\text { But, } 94=(25+69)=((25+16)+(69-16))=41+53,
$$

$e=16$ opts for this way of summation.

From above, we concluded until we expressed the sum of two prime numbers equal to an even number, we repeatedly do the above.

Theorem 1: Every even integer can be expressed as the difference of two prime numbers.

Proof: 
We know $2=5-3$, and difference of all twin primes; $4=7-3,11-7,17-$ $13,23-19 \ldots ; 6=11-5,17-11,23-17,29-23,37-31 \ldots ; 8=11-3,13-5$, $19-11 \ldots ; 10=13-3,17-7,23-13 \ldots ; \ldots$ but is it true for all even numbers? So, we try to prove every even integer can be expressed as the difference of two prime numbers by some ideological concepts.

Let $E$ be an even number and $O_{1}$ and $O_{2}$ are odd numbers.

We can express $E$ as difference of two odd numbers. i.e. $\quad E=O_{1}-O_{2}$.

Using the above facts, we can say all odd prime numbers are the members of odd numbers. i.e. $p \subset O$.

We know every prime number can be expressed as the sum of an odd number and even number. Also, we can express the prime number as the difference of an odd number and even number.

i.e. $o_{1}+e_{1}=p_{1} \in O_{1}$ and $o_{2}-e_{2}=p_{2} \in O_{2}$.

Hence, we can express $E=\left(O_{1}-O_{2}\right)=\left(\left(o_{1}+e_{1}\right)-\left(o_{2}-e_{2}\right)\right)=p_{1}-p_{2}$.

More precisely, we can express above as,

$$
\begin{aligned}
& E=\left(O_{1}-O_{2}\right)=\left(\left(o_{1}+e_{1}\right)-\left(o_{2}+e_{2}\right)\right)=p_{1}-p_{2} \\
& E=\left(O_{1}-O_{2}\right)=\left(\left(o_{1}-e_{1}\right)-\left(o_{2}-e_{2}\right)\right)=p_{1}-p_{2} \\
& E=\left(O_{1}-O_{2}\right)=\left(\left(o_{1}+e\right)-\left(o_{2}-e\right)\right)=p_{1}-p_{2}
\end{aligned}
$$

\section{Fibonacci Series}

$\begin{array}{lllllllllll}1 & 1 & 2 & 3 & 5 & 8 & 13 & 21 & 34 & 55 & 89\end{array}$

$\begin{array}{lllllllll}144 & 233 & 377 & 610 & 987 & 1597 & 2584 & \ldots\end{array}$

Various golden ratios of any two numbers of Fibonacci series:

$$
\begin{aligned}
& \varphi_{1}=\frac{f_{n+1}}{f_{n}}=\frac{f_{n}}{f_{n-1}}=\frac{f_{n-1}}{f_{n-2}}=\cdots=\frac{f_{n-r}}{f_{n-(r+1)}}=\varphi=2-1(2-\varphi) \\
& \varphi_{2}=\frac{f_{n+1}}{f_{n-1}}=\frac{f_{n}}{f_{n-2}}=\frac{f_{n-1}}{f_{n-3}}=\cdots=\frac{f_{n-r}}{f_{n-(r+2)}}=1+\varphi=3-1(2-\varphi) \\
& \varphi_{3}=\frac{f_{n+1}}{f_{n-2}}=\frac{f_{n}}{f_{n-3}}=\frac{f_{n-1}}{f_{n-4}}=\cdots=\frac{f_{n-r}}{f_{n-(r+3)}}=1+2 \varphi=5-2(2-\varphi) \\
& \varphi_{4}=\frac{f_{n+1}}{f_{n-3}}=\frac{f_{n}}{f_{n-4}}=\frac{f_{n-1}}{f_{n-5}}=\cdots=\frac{f_{n-r}}{f_{n-(r+4)}}=2+3 \varphi=8-3(2-\varphi) \\
& \varphi_{k+1}=\frac{f_{n+1}}{f_{n-k}}=\frac{f_{n}}{f_{n-k-1}}=\frac{f_{n-1}}{f_{n-k-2}}=\cdots=\frac{f_{n-r}}{f_{n-(r+k+1)}} \\
& =f_{k}+f_{k+1} \varphi=f_{k+3}-f_{k+1}(2-\varphi)
\end{aligned}
$$


Where 0.618 and 1 are the generators of the golden ratio Fibonacci series.

In this series also, we can get golden ratio.

$$
\begin{aligned}
& \text { i.e. } \frac{\Phi_{n+1}}{\Phi_{n}}=1.6180 \\
& \text { and } \frac{\Phi_{n}}{\Phi_{n+1}}=0.6180
\end{aligned}
$$

\section{Let We See Some Matrix Squares}

1) $4 \times 4$ Even Matrix Square

\begin{tabular}{|c|c|c|c|}
\hline $\mathbf{a}$ & $\mathbf{b}$ & $\mathbf{c}$ & $\mathbf{d}$ \\
\hline $\mathbf{e}$ & $\mathbf{f}$ & $\mathbf{g}$ & $\mathbf{h}$ \\
\hline $\mathbf{i}$ & $\mathbf{j}$ & $\mathbf{k}$ & $\mathbf{l}$ \\
\hline $\mathbf{m}$ & $\mathbf{n}$ & $\mathbf{o}$ & $\mathbf{p}$ \\
\hline
\end{tabular}

2) $5 \times 5$ Odd Matrix Square

\begin{tabular}{|c|c|c|c|c|}
\hline $\mathbf{a}$ & $\mathbf{b}$ & $\mathbf{c}$ & $\mathbf{d}$ & $\mathbf{e}$ \\
\hline $\mathbf{f}$ & $\mathbf{g}$ & $\mathbf{h}$ & $\mathbf{i}$ & $\mathbf{j}$ \\
\hline $\mathbf{k}$ & $\mathbf{I}$ & $\mathbf{m}$ & $\mathbf{n}$ & $\mathbf{o}$ \\
\hline $\mathbf{p}$ & $\mathbf{q}$ & $\mathbf{r}$ & $\mathbf{s}$ & $\mathbf{t}$ \\
\hline $\mathbf{u}$ & $\mathbf{v}$ & $\mathbf{w}$ & $\mathbf{x}$ & $\mathbf{y}$ \\
\hline
\end{tabular}

Let we walk on above matrix square, conditions are:

1) Our walking direction should be horizontally or vertically not diagonally. i.e. if we choose random place " $m$ " on $5 \times 5$ matrix square, we may go through leftward direction "l" or rightward direction " $\mathrm{n}$ " or upward direction " $\mathrm{h}$ " or downward direction " $r$ ". should not go through left upper diagonal "g", left lower diagonal " $q$ ", right upper diagonal " $\mathrm{i}$ ", and right lower diagonal " $\mathrm{s}$ ".

2) We should not return from the way once again which we went.

3) Randomly go any direction.

\subsection{Walking on Even $\times$ Even Matrix Square}

Theorem 2: If we start our walking from any box randomly on $E \times E$ matrix square, then we could return to our starting box once again.

Proof: 
Let " $E$ " be an even number, so we can construct $E \times E$ matrix square.

Now we start our walking at any arbitrary box in matrix square. That box would be one of the boxes of least even matrix square. That box has two directions if it would be a vertex, three directions if it would be a box on any sides and four directions if it would be an inner box.

Since $E \times E$ matrix square has even $E^{2}$ boxes.

If $E^{2}$ divided by 4 , then $E$ divided by 2 .

So we can divide even $E^{2}$ boxes by four boxes.

We get

$$
\frac{E^{2}}{4} \equiv 0(\bmod 4)
$$

This shows, $E^{2}$ boxes fully divided by four.

We can easily walk through four boxes which are given by $2 \times 2$ matrix square. From any point to that same point in $2 \times 2$ matrix square we could complete full walk.

So, we take $2 \times 2$ matrix square instead of $E \times E$ matrix square. Because $2 \times 2$ matrix square only full fills $E \times E$ matrix square.

It is enough to walk completely on $E \times E$ matrix square.

Ex5:

1) If $E=6$ we get $6^{2}$ matrix square, i.e. 36 boxes, $36=9 \times 4$ boxes.

2) If $E=8$ we get $8^{2}$ matrix square, i.e. 64 boxes, $64=16 \times 4$ boxes.

Walking on Odd $x$ Oddmatrix square

Theorem 3: If we start our walking from any box randomly on $O$ x $O$ matrix square, then we never return to our starting box once again.

Proof: $O \times O$ matrix square never gives $\frac{O^{2}}{4} \equiv 0(\bmod 4)$;

so, we never complete our walk

\subsection{Solution for Complete Walking on $\boldsymbol{O} \times \boldsymbol{O}$ Matrix Square}

Let we see some $O \times O$ matrix square.

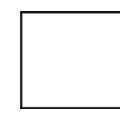

Odd $1 \times 1$ matrix square

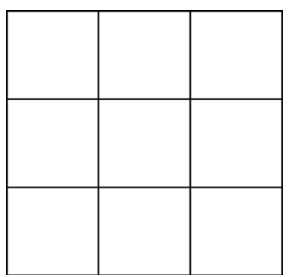

Odd $3 \times 3$ matrix square 


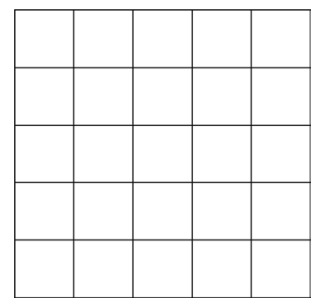

Odd $5 \times 5$ matrix square

Here after we call, odd $1 \times 1$ matrix square as first odd matrix square, odd $3 \times$ 3 matrix square as second odd matrix square, odd $5 \times 5$ matrix square as third odd matrix square.

Let we slightly modify odd $1 \times 1$ matrix square as, even $2 \times 2$ matrix.

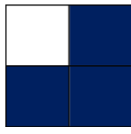

Let we slightly modify odd $3 \times 3$ matrix square as, even $4 \times 4$ matrix.

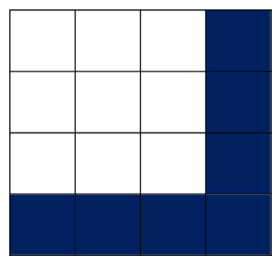

Let we slightly modify odd $5 \times 5$ matrix square as, even $6 \times 6$ matrix.

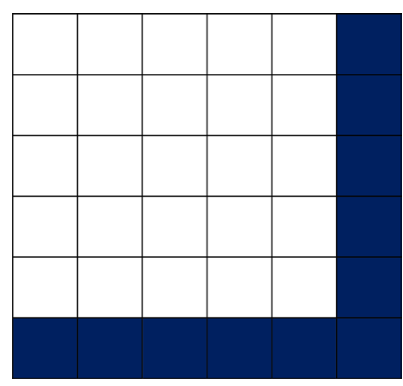

From the above we concluded,

$$
1+3=4 ; 9+7=16 ; 25+11=36 ; \cdots ; O^{2}+[3 k+1+(k-2)]=(O+1)^{2}=E^{2} .
$$

where $3 k+1$ is Collatz variant, $E$ is even number, $O$ is odd number, $k$ is $k^{\text {th }}$ matrix square, and $(k-2)$ is my variant for matrix square of Collatz sum, also

$$
k=\frac{O+1}{2} \text {. }
$$

Ex 6: Let 63 be a prime number, then $k=\frac{63+1}{2}=\frac{64}{2}=32$;

$$
63^{2}+[3 \times 32+1+(32-2)]=3969+[97+30]=3969+127=4096=64^{2} .
$$

Since $64 \times 64$ is a $32^{\text {th }}$ even matrix square. 
$\frac{64^{2}}{4} \equiv 0(\bmod 4)$. So we can easily complete our walking form starting box to starting box.

\subsection{Walking on $E \times E$ Matrix Square, Walking on $O \times O$ Matrix Square and above Results Shows How COLLATZ Conjecture Works}

$$
\frac{E^{2}}{4} \equiv 0(\bmod 4) \rightarrow \frac{E}{2} \equiv 0(\bmod 2) ;
$$

if $\frac{E}{2}=2^{t} \mid t \in I$ then we get $2^{t} \rightarrow 2^{t-1} \rightarrow 2^{t-2} \rightarrow \cdots \rightarrow 4 \rightarrow 2 \rightarrow 1$.

Else if $\frac{E}{2}=m o_{1} \mid m, o_{1} \in I$ then we do

$$
\frac{m o_{1}}{2} \equiv\left\{\begin{array}{l}
0(\bmod 2) \rightarrow \frac{m o_{1}}{2}=E_{1} \\
1(\bmod 2) \rightarrow 3 o_{1}+1 \rightarrow E_{1}
\end{array}\right.
$$

So, we get

if $\frac{E_{1}}{2}$ is $2^{t} \mid t \in I$ then we get $2^{t} \rightarrow 2^{t-1} \rightarrow 2^{t-2} \rightarrow \cdots \rightarrow 4 \rightarrow 2 \rightarrow 1$.

Else if $\frac{E_{2}}{2}=n o_{2} \mid n, o_{2} \in I$ then we do

$$
\frac{n o_{2}}{2} \equiv\left\{\begin{array}{l}
0(\bmod 2) \rightarrow \frac{n o_{2}}{2}=E_{2} \\
1(\bmod 2) \rightarrow 3 o_{2}+1 \rightarrow E_{2}
\end{array}\right.
$$

Do the above again and again,

finally, $E \rightarrow E_{1} \rightarrow E_{2} \rightarrow \cdots \rightarrow 4 \rightarrow 2 \rightarrow 1$.

But if $\frac{O^{2}}{4} \equiv o_{1}(\bmod 4) \rightarrow 3 O+1 \rightarrow E_{1}$ then

$$
\frac{E_{1}^{2}}{4} \equiv 0(\bmod 4) \rightarrow \frac{E_{1}}{2} \equiv 0(\bmod 2)
$$

if $\frac{E_{1}}{2}=2^{t} \mid t \in I$ then we get $2^{t} \rightarrow 2^{t-1} \rightarrow 2^{t-2} \rightarrow \cdots \rightarrow 4 \rightarrow 2 \rightarrow 1$.

Else if $\frac{E_{1}}{2}=m o_{1} \mid m, o_{1} \in I$ then we do

$$
\frac{m o_{1}}{2} \equiv\left\{\begin{array}{l}
0(\bmod 2) \rightarrow \frac{m o_{1}}{2}=E_{2} \\
1(\bmod 2) \rightarrow 3 o_{1}+1 \rightarrow E_{2}
\end{array}\right.
$$

So, we get

if $\frac{E_{2}}{2}=2^{t} \mid t \in I$ then we get $2^{t} \rightarrow 2^{t-1} \rightarrow 2^{t-2} \rightarrow \cdots \rightarrow 4 \rightarrow 2 \rightarrow 1$.

Else if $\frac{E_{2}}{2}=n o_{2} \mid n, o_{2} \in I$ then we do 


$$
\frac{n o_{2}}{2} \equiv\left\{\begin{array}{l}
0(\bmod 2) \rightarrow \frac{n o_{2}}{2}=E_{2} \\
1(\bmod 2 \rightarrow) 3 o_{2}+1 \rightarrow E_{2}
\end{array}\right.
$$

Do the above again and again,

finally, $E \rightarrow E_{1} \rightarrow E_{2} \rightarrow \cdots \rightarrow 4 \rightarrow 2 \rightarrow 1$.

Ex 7: Let 79 be an odd number then $k=\frac{79+1}{2}=40$;

$79 \times 79$ is $40^{\text {th }}$ odd matrix square.

$$
\begin{gathered}
\Rightarrow \frac{79^{2}}{4} \equiv 1(\bmod 4) \rightarrow 3 \times 79+1=238 \\
\Rightarrow \frac{238}{2} \equiv 0(\bmod 2) \rightarrow 238=2 \times 119 \\
\Rightarrow 79 \rightarrow 238 \rightarrow 119 \rightarrow 358 \rightarrow 179 \rightarrow 538 \rightarrow 269 \rightarrow 808 \rightarrow 404 \rightarrow 202 \rightarrow 101 \\
\rightarrow 304 \rightarrow 152 \rightarrow 76 \rightarrow 38 \rightarrow 19 \rightarrow 58 \rightarrow 29 \rightarrow 88 \rightarrow 44 \rightarrow 22 \rightarrow 11 \rightarrow 34 \\
\rightarrow 17 \rightarrow 52 \rightarrow 26 \rightarrow 26 \rightarrow 13 \rightarrow 40 \rightarrow 20 \rightarrow 10 \rightarrow 5 \rightarrow 16 \rightarrow 8 \rightarrow 4 \rightarrow 2 \rightarrow 1 .
\end{gathered}
$$

Finally we attained 1 . Since 1 is a starting number of all natural numbers. We once again returned to 1 .

But our matrix square formulaeasily confirms the above calculation.

My matrix square formula for Collatz sum

$$
\begin{aligned}
& =O^{2}+[3 k+1+(k-2)]=(O+1)^{2}=E^{2} \\
& =79^{2}+[3 \times 40+1+(40-2)] \\
& =6241+121+38=6400=80^{2}
\end{aligned}
$$

$\Rightarrow \frac{80^{2}}{4} \equiv 0(\bmod 4)$, it shows 79 converged to 1 . And $80 \times 80$ is the $40^{\text {th }}$ even matrix square.

In this proof we omit $1 \times 1$ square matrix as the first odd matrix. We consider $3 \times 3$ square matrix as the first odd matrix square, $5 \times 5$ square matrix as the second odd matrix square, $7 \times 7$ square matrix as the third odd matrix square...

Let we slightly modify odd $3 \times 3$ matrix square as, even $2 \times 2$ matrix.

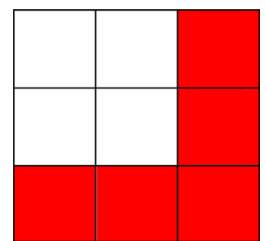

Let we slightly modify odd $5 \times 5$ matrix square as, even $4 \times 4$ matrix.

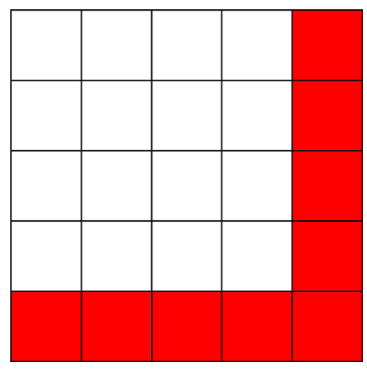


From the above we concluded,

$$
1-(-3)=4 ; 9-5=4 ; 25-9=16 ; \cdots ; O^{2}-[3 k-1+(k+2)]=(O-1)^{2}=E^{2} \text {. }
$$

Where $3 k-1$ is Tao variant, $E$ is even number, $O$ is odd number, $k$ is $k^{\text {th }}$ matrix square, and $(k+2)$ is my variant for matrix square of Tao sum, also

$$
k=\frac{O-1}{2} .
$$

9.4. Walking on $E \times E$ matrix Square, Walking on $O \times O$ Matrix Square and above Results Shows How COLLATZ-TAO Conjecture Works

$$
\frac{E^{2}}{4} \equiv 0(\bmod 4) \rightarrow \frac{E}{2} \equiv 0(\bmod 2) ;
$$

if $\frac{E}{2}=2^{t} \mid t \in I$ then we get $2^{t} \rightarrow 2^{t-1} \rightarrow 2^{t-2} \rightarrow \cdots \rightarrow 4 \rightarrow 2 \rightarrow 1$.

Else if $\frac{E}{2}=m o_{1} \mid m, o_{1} \in I$ then we do

$$
\frac{m o_{1}}{2} \equiv\left\{\begin{array}{l}
0(\bmod 2) \rightarrow \frac{m o_{1}}{2}=E_{1} \\
1(\bmod 2) \rightarrow 3 o_{1}-1 \rightarrow E_{1}
\end{array}\right.
$$

So, we get if $\frac{E_{1}}{2}$ is $2^{t} \mid t \in I$ then we get

$2^{t} \rightarrow 2^{t-1} \rightarrow 2^{t-2} \rightarrow \cdots \rightarrow 4 \rightarrow 2 \rightarrow 1$.

Else if $\frac{E_{2}}{2}=n o_{2} \mid n, o_{2} \in I$ then we do

$$
\frac{n o_{2}}{2} \equiv\left\{\begin{array}{l}
0 \bmod 2 \rightarrow \frac{n o_{2}}{2}=E_{2} \\
1 \bmod 2 \rightarrow 3 o_{2}-1 \rightarrow E_{2}
\end{array}\right.
$$

Do the above again and again,

finally, $E \rightarrow E_{1} \rightarrow E_{2} \rightarrow \cdots \rightarrow 4 \rightarrow 2 \rightarrow 1$.

But if $\frac{O^{2}}{4} \equiv o_{1}(\bmod 4) \rightarrow 3 O-1 \rightarrow E_{1}$ then

$$
\frac{E_{1}^{2}}{4} \equiv 0(\bmod 4) \rightarrow \frac{E_{1}}{2} \equiv 0(\bmod 2)
$$

If $\frac{E_{1}}{2}=2^{t} \mid t \in I$ then we get $2^{t} \rightarrow 2^{t-1} \rightarrow 2^{t-2} \rightarrow \cdots \rightarrow 4 \rightarrow 2 \rightarrow 1$.

Else if $\frac{E_{1}}{2}=m o_{1} \mid m, o_{1} \in I$ then we do

$$
\frac{m o_{1}}{2} \equiv\left\{\begin{array}{l}
0(\bmod 2) \rightarrow \frac{m o_{1}}{2}=E_{2} \\
1(\bmod 2) \rightarrow 3 o_{1}-1 \rightarrow E_{2}
\end{array}\right.
$$

So, we get

if $\frac{E_{2}}{2}=2^{t} \mid t \in I$ then we get $2^{t} \rightarrow 2^{t-1} \rightarrow 2^{t-2} \rightarrow \cdots \rightarrow 4 \rightarrow 2 \rightarrow 1$. 
Else if $\frac{E_{2}}{2}=n o_{2} \mid n, o_{2} \in I$ then we do

$$
\frac{n o_{2}}{2} \equiv\left\{\begin{array}{l}
0(\bmod 2) \rightarrow \frac{n o_{2}}{2}=E_{2} \\
1(\bmod 2) \rightarrow 3 o_{2}-1 \rightarrow E_{2}
\end{array}\right.
$$

Do the above again and again,

finally, $O \rightarrow E_{1} \rightarrow E_{2} \rightarrow \cdots \rightarrow 4 \rightarrow 2 \rightarrow 1$.

Ex 8: Let 79 be an odd number then $k=\frac{79-1}{2}=39$;

$79 \times 79$ is $39^{\text {th }}$ odd matrix square. Since in this sum we omitted $1 \times 1$ as a first odd matrix square. $\Rightarrow \frac{79^{2}}{4} \equiv 1(\bmod 4) \rightarrow 3 \times 79-1=236$

$$
\begin{gathered}
\quad \Rightarrow \frac{236}{2} \equiv 0(\bmod 2) \rightarrow 236=2 \times 118 \\
\Rightarrow 79 \rightarrow 236 \rightarrow 118 \rightarrow 59 \rightarrow 176 \rightarrow 88 \rightarrow 44 \rightarrow 22 \\
\rightarrow 11 \rightarrow 32 \rightarrow 16 \rightarrow 8 \rightarrow 4 \rightarrow 2 \rightarrow 1 .
\end{gathered}
$$

Finally we attained 1 . Since 1 is a starting number of all natural numbers. We once again returned to 1 .

But our matrix square formula easily confirms the above calculation.

$$
\begin{gathered}
\text { My matrix square formula for Tao sum } \\
=O^{2}-[3 k-1+(k+2)]=(O-1)^{2}=E^{2} \\
=79^{2}-[3 \times 39-1+(40+2)] \\
=6241-116-41=6084=78^{2} \\
\Rightarrow \frac{78^{2}}{4} \equiv 0(\bmod 4)
\end{gathered}
$$

it shows 79 converged to 1 . And $78 \times 78$ is the $39^{\text {th }}$ even matrix square.

\section{Conclusions}

1) Plane is a two dimensional volume, Binomial expression has two elements. This is the reason, we could generalize Euler's formula $V-E+F=2$ for planer graphs by Binomial expressions. Similarly, we can say, an edge draws between two vertices in planer graphs, so only two is acted as the multiplicative factor of binomial expression.

2) Generally we concluded that, number of vertices is act as the multiplicative factor of binomial expression in $\mathrm{n}^{\text {th }}$ cubic graphs.

$3)$ There are so many numbers that would not be the value of any $\varphi(n)$.

4) Pair of twin prime is $6 x-1$ and $6 x+1$. So only six numbers being existed between any two consecutive twin prime pairs and $\mathrm{d}(6 x-1,6 x+1)=2$.

5) Other than two all prime numbers are proper sub-set of odd numbers. We already know the sum of any two odd numbers is an even number. So we concluded that, every even integer greater than 2 can be written as the sum of two primes. 
6) Every even integer can be written as the difference between two primes.

7) All the Fibonacci series like series converged to the golden ratio, even the series elements are golden ratio elements.

8) We concluded matrix square concept is parallel proof for Collatz conjecture and Collatz-Tao conjecture.

\section{Conflicts of Interest}

The author declares no conflicts of interest regarding the publication of this paper.

\section{References}

[1] https://en.wikipedia.org/wiki/Pascal_triangle

[2] https://en.wikipedia.org/wiki/Planar_graph

[3] https://en.wikipedia.org/wiki/Euler totient_function

[4] https://en.wikipedia.org/wiki/Twin_prime

[5] https://en.wikipedia.org/wiki/Golden_ratio

[6] https://en.wikipedia.org/wiki/Goldbach_conjecture

[7] https://en.wikipedia.org/wiki/Collatz_conjecture

[8] Rangasamy, B.P. (2020) Balasubramani Prema Rangasamy-One Travel from Vertex to Vertex via Edge on Face. London Journals Press, Theale.

[9] Rangasamy, B.P. (2020) Balasubramani Prema Rangasamy-Conjecturing with Conjectures. Global Journal of Science Frontier Research, 20, 69-75. 